Progress in Flight Physics 5 (2013) 349-362

DOI: $10.1051 /$ eucass/201305349

(C) Owned by the authors, published by EDP Sciences, 2013

\title{
SUPERSONIC LAMINAR SEPARATED FLOW STRUCTURE AT A RAMP FOR A FREE-STREAM MACH NUMBER OF 6
}

\author{
V. I. Zapryagaev ${ }^{1}$, I. N. Kavun ${ }^{1}$, and I. I. Lipatov ${ }^{2}$ \\ ${ }^{1}$ Khristianovich Institute of Theoretical and Applied Mechanics \\ 4/1 Institutskaya Str., Novosibirsk 630090, Russia \\ ${ }^{2}$ TsAGI \\ 1 Zhukovsky Str., Zhukovsky, Moscow Region 140180, Russia
}

\begin{abstract}
A three-dimensional (3D) structure of a separated flow in a compression corner at a free-stream Mach number $\mathrm{M}=6$ is studied. The model is a flat plate with an almost sharp leading edge on which a $30^{\circ}$ ramp is mounted. The spanwise size of the model is equal to the length of the plate from the leading edge to the ramp. The angle of attack is varied from $0^{\circ}$ to $15^{\circ}$. The shock wave structure of the flow near the model is shown. The existence of a thin high total pressure layer (dynamic layer) is found. The dynamic layer is located above the boundary layer on the ramp, downstream from the reattachment line. The existence streamwise vortices near the reattachment line is shown. The measured spectra of the wall pressure fluctuations in the reattachment region are presented.
\end{abstract}

\section{NOMENCLATURE}

$c_{p}=\left(p-p_{\infty}\right) / q$ pressure coefficient (here, $p$ is the wall pressure)

$d \quad$ width of model, $\mathrm{m}$

$d_{\mathrm{tr}} \quad$ diameter of the pressure fluctuations transducer, $\mathrm{m}$

$f \quad$ frequency, $\mathrm{Hz}$

$l \quad$ lengthwise coordinate, $\mathrm{m}$

$L \quad$ length of model plate, $\mathrm{m}$

M Mach number

$\mathrm{M}_{\infty} \quad$ free-stream Mach number

$p \quad$ pressure, $\mathrm{Pa}$

$p^{\prime} \quad$ root-mean-square value of pressure fluctuations, $\mathrm{Pa}$

$p_{\operatorname{tr}} \quad$ wall pressure at the location of the pressure fluctuations transducer, $\mathrm{Pa}$ 


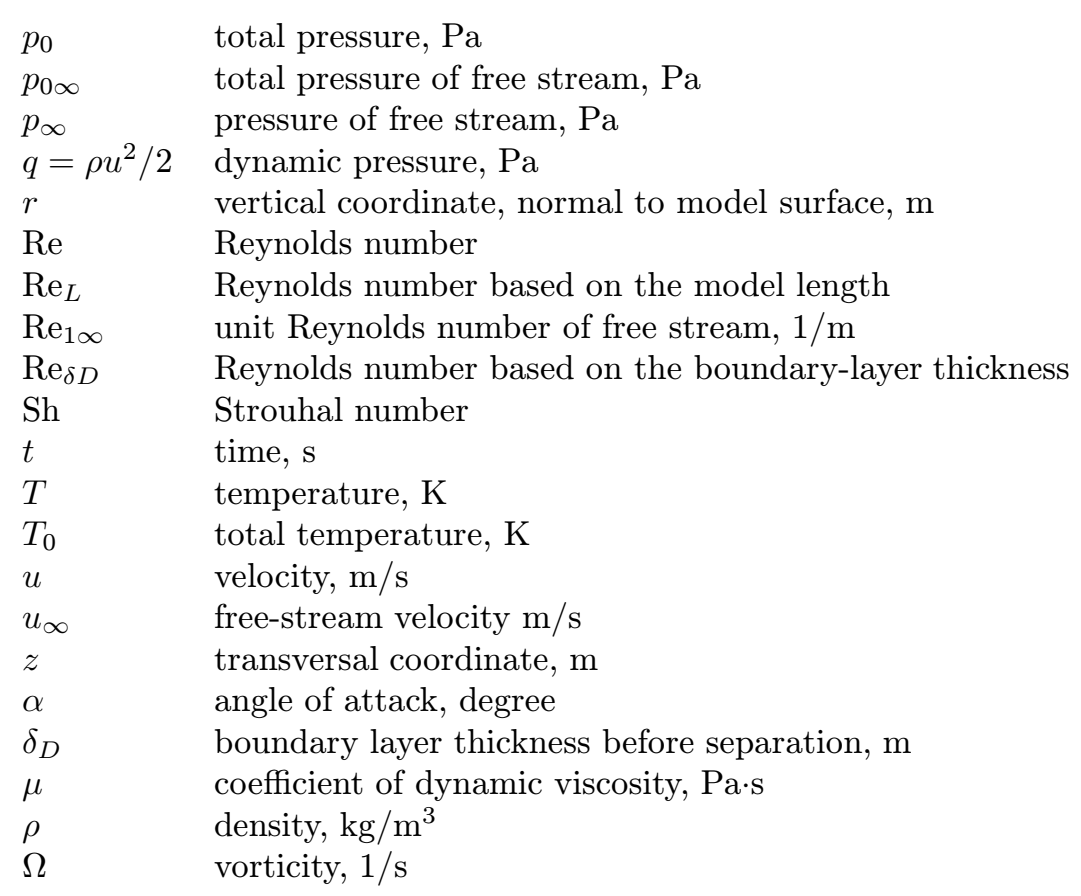

\section{INTRODUCTION}

A supersonic flow with a separation region near the ramp is a canonical problem involving the interaction of shock waves with the boundary layer (see, e.g. [1$7]$ ). At a high free-stream Mach number, the shock waves are formed over the separation zone and give rise to a complex shock-wave structure that affects the downstream evolution of flow characteristics behind the shear-layer reattachment line. The model size in the transverse direction is limited, so the flow structure in the separation zone is always $3 \mathrm{D}$; this circumstance additionally complicates the flow description. The goal of the present investigation is to clarify the $3 \mathrm{D}$ flow structure effects on the distribution of the mean and fluctuating flow characteristics near the model surface. Results of a complex experimental study of the separated flow structure at high supersonic flow velocities are also required for verification of numerical calculations.

In the present paper, results of an experimental and numerical study of the 3 D laminar separated flow at a ramp for various angles of attack are reported. The model was a plate with a sharp leading edge on which a 30 degree ramp was mounted (Fig. 1a). The study comprised a Schlieren and oil visualization of the flow, measurement of the wall pressure, and registration of wall-pressure fluctuations at a point located on the ramp surface near the flow reattachment 


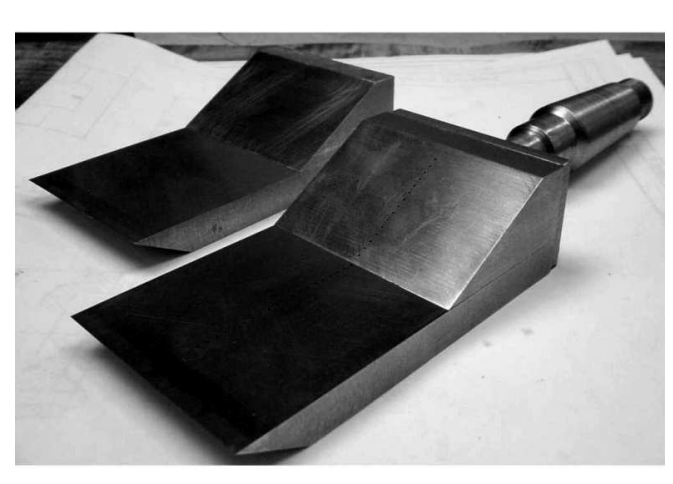

(a)

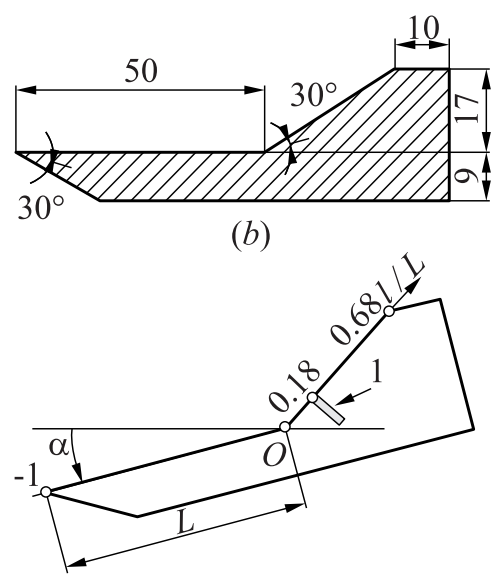

(c)

Figure 1 Compression-corner model. Dimensions are in millimeters

line. The experimental data are supplemented with results of a numerical study of the $3 \mathrm{D}$ structure of the flow.

\section{EXPERIMENTAL AND NUMERICAL CALCULATION TECHNIQUE}

\section{$2.1 \quad$ Experimental Technique}

The experiment was carried out in the T-326 wind tunnel of the Khristianovich Institute of Theoretical and Applied Mechanics of the Siberian Branch of the Russian Academy of Sciences (ITAM SB RAS). The wind tunnel is equipped with a test section made as the Eiffel chamber $0.87 \mathrm{~m}$ long, $1.18 \mathrm{~m}$ high, and $0.83 \mathrm{~m}$ wide. The nozzle exit diameter is $0.2 \mathrm{~m}$. The range of Mach numbers is $\mathrm{M}_{\infty}=6-4$. The range of unit Reynolds numbers is $\operatorname{Re}_{1 \infty}=(0.6-70) \cdot 10^{6} \mathrm{~m}^{-1}$. The plenum chamber pressure is $p_{\leq} 10 \mathrm{MPa}$, and the temperature in the chamber is $T_{0} \leq 1500 \mathrm{~K}$. The test time is $\sim 25 \mathrm{~min}$. The Mach number nonuniformity in the test section is $\leq 0.5 \%$. The wind tunnel is started without the model in the hypersonic flow, but later the model is inserted into the flow by using a special electromechanical device. The model insertion time is approximately $1 \mathrm{~s}$.

For carrying out the experiments, two identical models of the compression corner were manufactured (see Fig. 1a); each of the models was a plate with an almost sharp leading edge on which a ramp was mounted. One model was used for tap pressure measurements and another one was used for pressure fluctuations 
measuring. The leading edge was slightly blunt. The rounding radius of the leading edge of the plate was about $30 \mu \mathrm{m}$.

The model sizes are given in millimeters in the sketch (Fig. 1b). The spanwise size (width) of the model was $d=50 \mathrm{~mm}$. For convenience of data analysis, a dimensionless coordinate system $(0, l / L)$ located along the upper surface of the model in its midsection was introduced. In this coordinate system, the origin $O$ is located at the line of intersection of the horizontal surface of the plate and the ramp sloping surface (Fig. 1c). The plate length $L$ was used to obtain the dimensionless linear coordinate $l / L$. The value $l / L=-1$ refers to the tip of the sharp leading edge of the plate. The model was mounted by using a sting in the mechanism for changing the angle of attack ( $\alpha$-mechanism) of the T-326 wind tunnel. The model could be installed at different angles of attack $\alpha$. The experiments were performed for $\alpha=0^{\circ}, 5^{\circ}, 10^{\circ}$, and $15^{\circ}$. The inaccuracy in setting the angle $\alpha$ was within $0.1^{\circ}$. During the experiments, the deflection of the model by the approaching flow was smaller than $0.1^{\circ}$ (the measurements were performed by using a special sensor in the $\alpha$-mechanism); therefore, no subsequent regard will be given for this deflection.

The following wind tunnel gasdynamic parameters were used: mean Mach number of the flow at the model location $\mathrm{M}_{\infty}=6.01$, the free-stream total pressure $p_{0 \infty}=9.73 \cdot 10^{5} \mathrm{~Pa}$, and the free-stream total temperature $T_{0 \infty}=380 \mathrm{~K}$. The Reynolds number based on the model length was $\operatorname{Re}_{L}=6 \cdot 10^{5}$ ( $L$ is the length of the horizontal surface of the model, $L=50 \mathrm{~mm}$ ). Condensation of oxygen and nitrogen is absent as a result of a small length of the divergent part of the supersonic nozzle (smaller than $1 \mathrm{~m}$ ). The time of the model presence in the hypersonic flow for measurements is $3-7 \mathrm{~min}$. The model temperature increases from 290 to $320 \mathrm{~K}$ during the test time.

The wall pressure was measured by TDM-A-1.0, TDM-A-1.6, and SIEMENS HP-34970A pressure transducers. The pressure taps were located in the region $-0.81 \leq l / L \leq 0.6$ with a step $l / L=0.04$.

The pressure fluctuations $p^{\prime}(t)$ were measured with an I-2141 piezoelectric transducer (see Fig. 1c, marked by 1). The transducer was flush-mounted with the ramp sloping surface at the model centerline at the point $l / L=0.18$. The outer diameter of the transducer was $d_{\mathrm{tr}}=4 \mathrm{~mm}$.

Schlieren visualization of the flow was performed using an IAB-451 shadow instrument. A horizontal knife was used, which allowed capturing the vertical gradient of the gas density. Video recording was performed with a Videoscan$285 /$ P-USB digital video camera at an exposure time of $0.5 \mathrm{~ms}$.

\subsection{Numerical Calculation Technique}

Numerical calculations were performed using the Fluent software. The calculations were performed for the angle of attack $\alpha=0^{\circ}$. In the calculations, a 
compression-corner flow was modeled, with the geometry and sizes being the same as in the experiment. The domain of calculation comprised about 9 million cells. Near the model wall, the calculation grid was refined. In the refined grid, the thickness of the boundary layer near the flow separation point at the longitudinal symmetry axis of the model contained about fifty cells.

Three-dimensional laminar Navier-Stokes equations were solved. Those equations were linearized in an explicit form by a second-order upwind scheme. Though the flow at the reattachment region and inside the reverse flow region can be transitional or turbulent, this approach ensures a satisfactory agreement between the calculated and experimental data. The calculated ambient medium was a perfect gas. The gas viscosity was calculated by the Sutherland formula.

The decrease in temperature at the horizontal plate for the laminar boundary layer for a stationary flow is $321 \mathrm{~K}$. This temperature value is close to the model temperature after the experiment. Therefore, the heat flux between the model wall and the flow was accepted to be equal to zero (adiabatic wall condition).

\section{RESULTS}

\subsection{Mean Shock-Wave Structure of the Compression-Corner Flow at Different Angles of Attack}

The Schlieren photographs and schemes of the compression-corner flows for $\alpha$ $=0^{\circ}, 5^{\circ}, 10^{\circ}$, and $15^{\circ}$ are shown in Fig. 2. On the diagrams, the shock waves are shown by the solid lines and are denoted by the letter $C$; the expansion waves are shown by the dashed lines and are denoted by the letter $E$. The shear layers are shown by the dotted lines and are denoted by the abbreviation $S L$. The reverse flow region is denoted by the letter $Z$. Below in the text, the shock $C_{1}$ is called the leading shock; the shock $C_{4}$ is the trailing shock; the shock $C_{2}$ is the separation shock; and the shock $C_{3}$ is the reattachment shock. The shear layer enclosing the separation zone $Z$ is denoted by $S L_{1}$. The separation point (line) is denoted by the letter $D$, and the reattachment point (line) is denoted by $R$.

The calculated values of the Mach number in the flow region above the boundary layer before the separation point (at the point $D$, i. e., in the region between the shocks $C_{1}$ and $C_{2}$ ) for $\alpha=0^{\circ}, 5^{\circ}, 1^{\circ}$, and $15^{\circ}$ are $\mathrm{M}_{D}=6.01,5.32$, 4.64 , and 3.99, respectively. The boundary layer thickness before separation $\delta_{D}$ (point $D$ ) was estimated from the schlieren photographs; in various experiments, this thickness was $\delta_{D} \sim 0.7-1 \mathrm{~mm}$, and the Reynolds-number value based on the boundary-layer thickness was $\operatorname{Re}_{\delta D} \sim(10-15) \cdot 10^{3}$. 

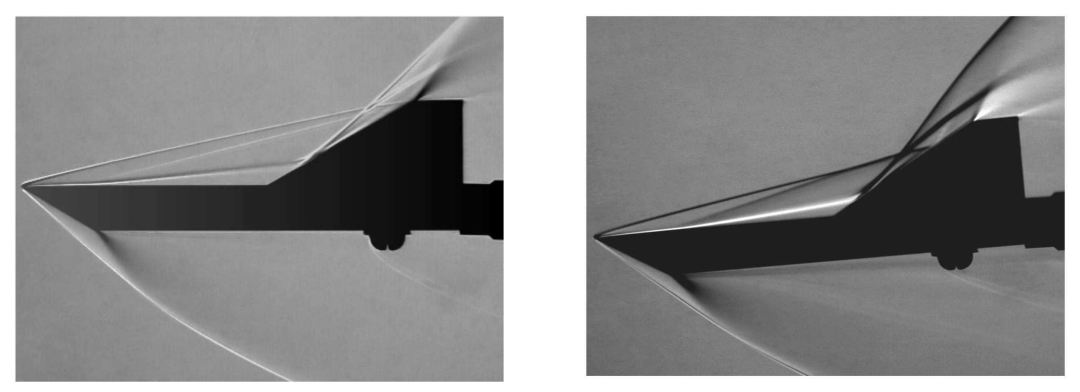

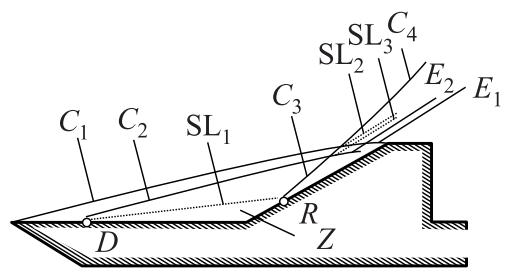

(a)
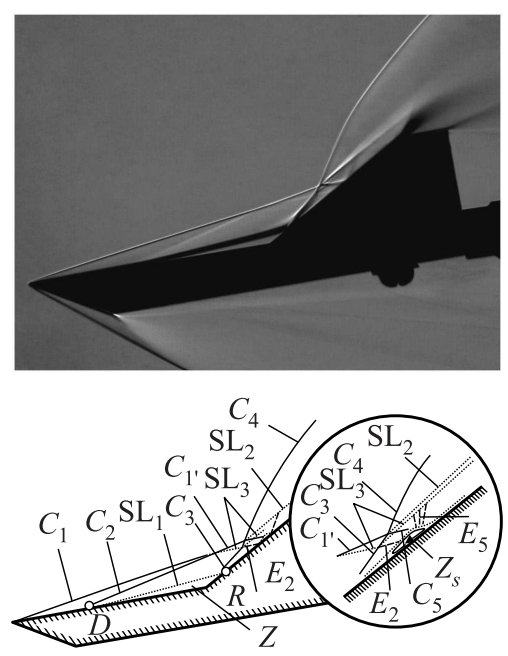

(c)

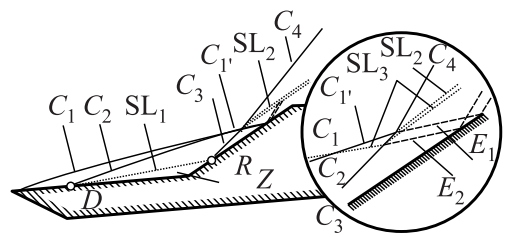

(b)
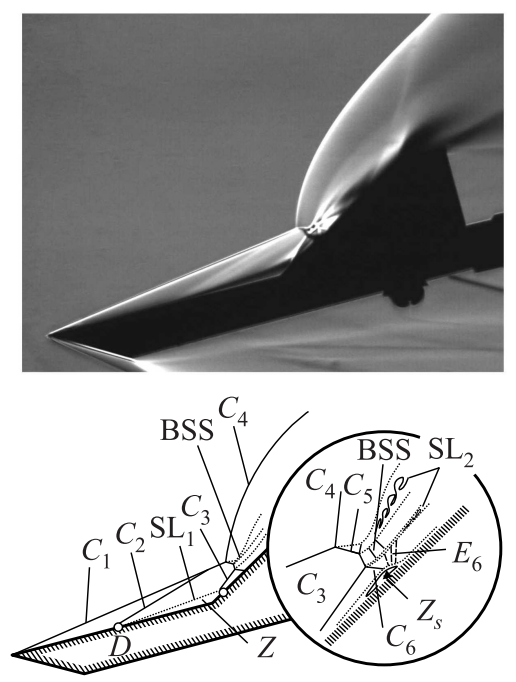

(d)

Figure 2 Schlieren photographs and explanatory diagrams of the compression-corner flows for $\alpha=0^{\circ}(a) ; 5^{\circ}(b) ; 10^{\circ}(c)$; and $\alpha=15^{\circ}(c)$ 
At the zero angle of attack, $\alpha=0^{\circ}$, the shocks $C_{1}$ and $C_{2}$ interact with the shock $C_{3}$ and form the shock $C_{4}$. The shock $C_{3}$ closes the separation zone. The shear layers $\mathrm{SL}_{2}$ and $\mathrm{SL}_{3}$ and the expansion waves $E_{1}$ and $E_{2}$ correspond to the flow parameters in the region behind the shock $C_{3}$ and the flow parameters in the region behind the shock $C_{4}$. This shock wave interaction corresponds to VI type configuration according to Edney's classification [8].

For higher angles of attack $\left(\alpha=5^{\circ}\right.$ and $\left.10^{\circ}\right)$, the shocks $C_{1}$ and $C_{2}$ interact and generate the shock wave $C_{1}^{\prime}$ and the shear layer $\mathrm{SL}_{3}$. The interaction of the shear layer $\mathrm{SL}_{3}$ with the shock $C_{3}$ results in shear layer deflection and formation of the expansion wave $E_{2}$. The interaction of the shocks $C_{1^{\prime}}$ and $C_{3}$ results in the formation of the shock $C_{4}$, the shear layer $\mathrm{SL}_{2}$, and either the expansion wave $E_{1}\left(\alpha=5^{\circ}\right)$ or the shock $C_{5}$ and the expansion wave $E_{5}\left(\alpha=10^{\circ}\right)$.

At the model angle $\alpha=15^{\circ}$, the shocks $C_{1}, C_{2}$, and $C_{3}$ interact with each other and form an intricate shock-wave structure. The interaction of the shocks $C_{3}$ and $C_{5}$ results in the formation of a special shock-wave structure, which is similar to the barrel shock-wave structure. This shock-wave structure can appear in a plane underexpanded jet and is directed in the downstream direction parallel to the ramp surface (this barrel shock-wave structure is denoted by BSS on the diagram). Between the barrel shock-wave structure and the ramp surface, the shock $C_{6}$ is formed. The interaction of the shock $C_{6}$ with the ramp wall results in the formation of a local microseparation region; so, one can see a new expansion wave $E_{6}$ (Fig. $2 b$ ). It is a typical picture of the boundary layer/shock wave interaction, see the paper of J. Delery in [7]. The described interaction results in the formation of strongly turbulent (upper and lower) shear layers $\mathrm{SL}_{2}$, also seen in the photograph. This shock-wave configuration corresponds to the $\mathrm{V}$ type of shock-wave interaction [8].

The photographs and flow diagrams for $\alpha=0^{\circ}$ and $15^{\circ}$ are similar to those previously reported for the flow with the free-stream Mach number $\mathrm{M}_{\infty}=7$ in $[5]$.

The data on the distribution of the pressure coefficient $c_{p}$ as a function of the coordinate $l / L$ along the line of intersection of the model upper surface with the longitudinal axis of symmetry are shown in Fig. 3. The initial point $l / L=0$ refers to the beginning of a ramp surface. The regions $l / L<0$ and $l / L>0$ refer, respectively, to the plate and ramp surface.

Qualitatively, the data on the distribution of the wall pressure agree with the visualization data. The pressure rises due to the compression shocks $C_{5}$ and $C_{6}$, and the pressure falls due to the expansion waves $E_{1}, E_{2}, E_{5}$, and $E_{6}$, observed in the photographs taken at various angles $\alpha$. A possible cause (see [9]) for the registration of the shock $C_{5}$ and the subsequent expansion wave $E_{5}$ (for $\alpha=10^{\circ}$ ) is the presence of a local separation region $Z_{s}$ (see the diagram in Fig. $2 c$ ) formed near the line of incidence of the shock $C_{5}$ onto the ramp surface. Similarly, at $\alpha=15^{\circ}$, such a region $Z_{s}$ is also formed at the place of incidence of the shock $C_{6}$. 


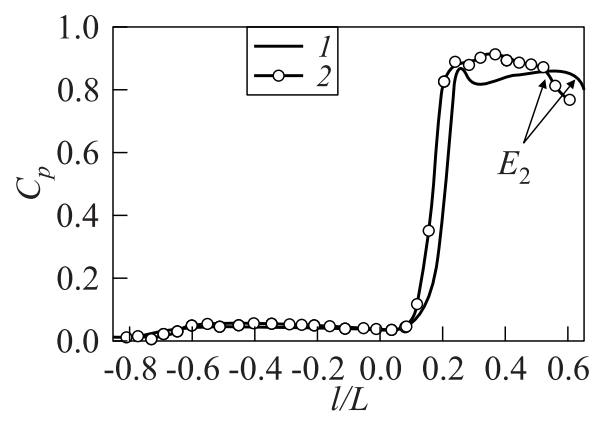

(a)

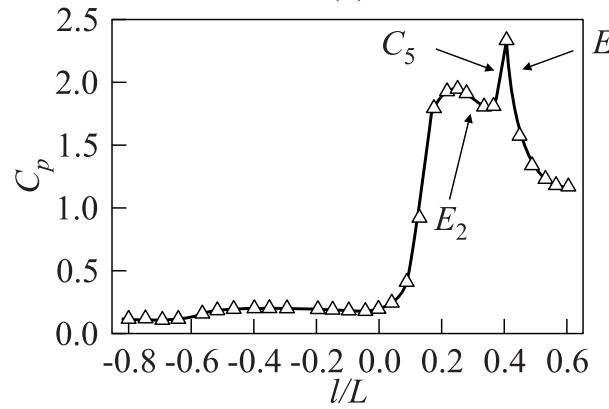

(c)

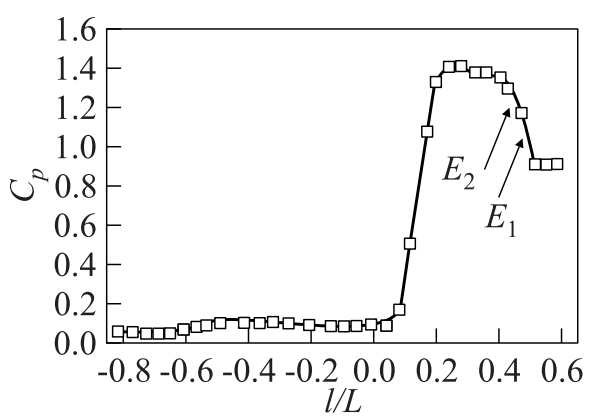

(b)

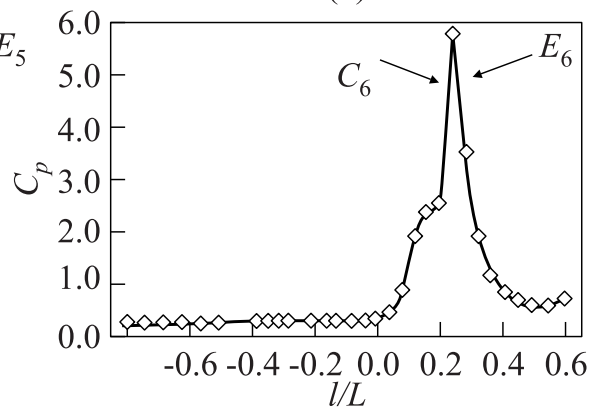

(d)

Figure 3 Distribution of the wall pressure over the longitudinal symmetry axis of the model for different angles $\alpha$ : (a) $0^{\circ}(1$ - numerical calculation; and 2 - experiment); (b) $5^{\circ} ;(c) 10^{\circ}$; and $(d) 15^{\circ}$

\subsection{High-Pressure Layer Behind the Shear Layer Reattachment Line}

Based on the numerical data, below an analysis of the flow structure near the reattachment line $R$ is given. An interesting feature here is the existence of a high-pressure layer or dynamic layer (DL) formed behind the reattachment line $R$ of the shear layer $\mathrm{SL}_{1}$ in the region above the boundary layer and propagating in the downstream direction (Fig. 4). Here, the total pressure in the layer DL is much higher than the pressure in the layers above or below this layer. Figure $4 a$ shows the contour lines of the calculated field of the total pressure $p_{0}$ normalized by the free-stream total pressure $p_{0 \infty}$ in the longitudinal symmetry plane of the model. At the right side of the figure, the diagrams of the total pressure $p_{0} / p_{0 \infty}$ in cross sections 1 and 2 (in front of and behind the shear layer reattachment point $R$, respectively) are shown. Figure $4 b$ contains a diagram that explains the physical mechanism of high pressure layer formation. 


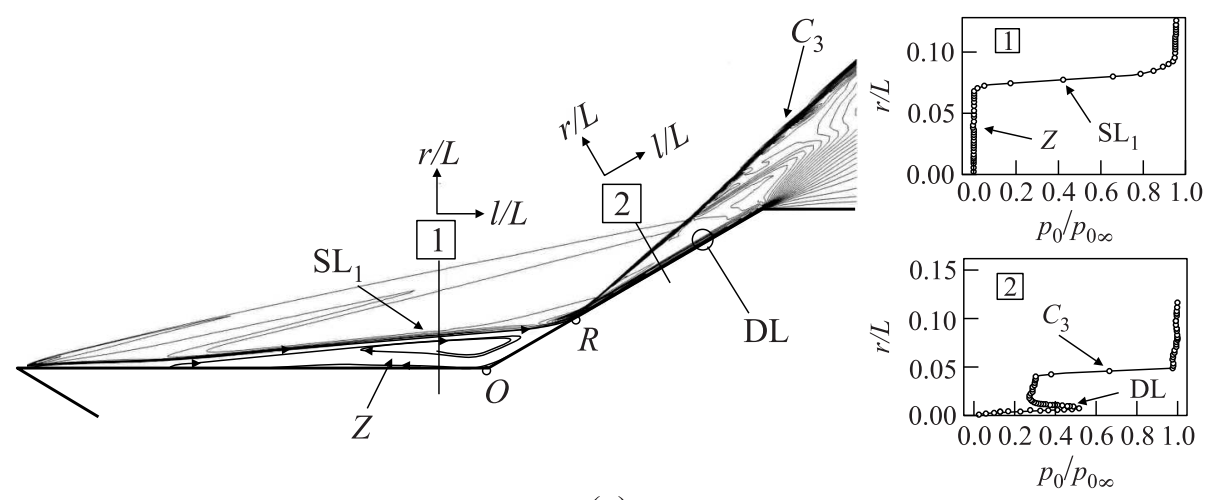

(a)

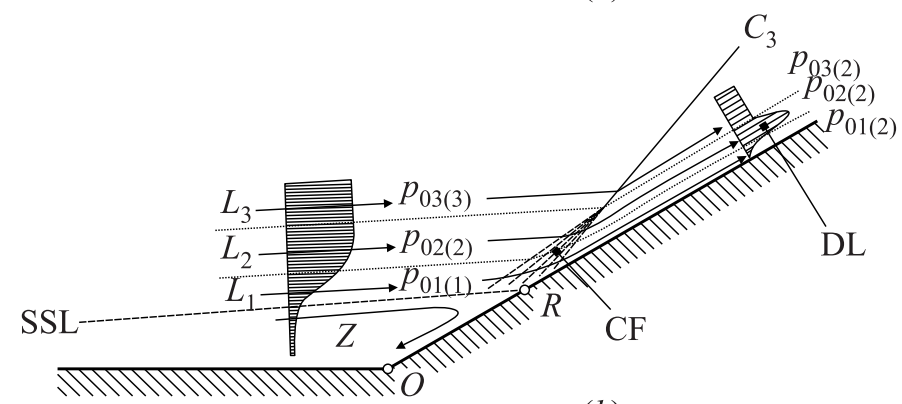

(b)

Figure 4 High-pressure layer DL behind the flow reattachment line

The gas stream in the region above the streamline SSL (this streamline arrives at the reattachment point $R$ ) can be divided into three layers: $L_{1}$ is the subsonic layer in the shear flow $\mathrm{SL}_{1}, L_{2}$ is the supersonic layer in the shear flow $\mathrm{SL}_{1}$, and $L_{3}$ is the supersonic flow that moves over the layer $\mathrm{SL}_{1}$. The loss of the total pressure in the layer $L_{1}$ due to friction forces is much greater than the loss of the total pressure in the layer $L_{2}$ (which is low) and in the layer $L_{3}$; hence, the total pressure $p_{01(1)}$ in cross section $1(l / L=-0.1)$ in the layer $L_{1}$ is much lower than the total pressures $p_{02(1)}$ and $p_{03(1)}$ in the layers $L_{2}$ and $L_{3}$. In the course of stream deflection near the point $R$, a compression fan $\mathrm{CF}$ is formed, which transforms into the shock wave $C_{3}$ at some distance from the model surface. The loss of the total pressure in the shock $C_{3}$ in the layer $L_{3}$ is greater than the loss of the total pressure in the layer $L_{2}$ (the flow has turned in the compression fan $\mathrm{CF}$ ). As a result, the total pressure in the layer $L_{3}$ becomes lower than that in the layer $L_{2}$. Thus, the total pressure $p_{02(2)}$ in the layer $L_{2}$ is greater than the pressures $p_{01(2)}$ and $p_{03(2)}$ in the layers $L_{1}$ and $L_{3}$ in section $2(l / L=0.58)$. This layer $L_{3}$ with a high total pressure can be called the high-pressure layer or the dynamic pressure layer DL. 
Owing to the existence of the high-pressure layer DL, the gas density and the Mach number in this layer become higher, but the static temperature is lower than the respective quantities in the boundary layer on the ramp surface and in the free stream in the flow region above the ramp.

\subsection{Streamwise Vortices Near the Model Surface}

The oil visualization (Fig. $5 a, \alpha=0^{\circ}$ ) shows a $3 \mathrm{D}$ pattern of the flow in the separation region $Z$. The curved flow separation line $D$, the curved flow reattachment line $R$, and the reverse flow region $Z$ are clearly seen. Also, the presence of streamwise vortices spreading from the flow reattachment line $R$ both inward the separation zone and in the downstream direction is noteworthy (here, alternation of dark and light strips oriented along the free-stream direction is seen). The diagram in Fig. $5 b$ was plotted using the calculated data for the instantaneous distribution of the shear stress on the model surface. The calculated lines of the flow separation $D$, the reattachment $R$, and the reversed flow region are shown. The shear stress picture shows a $3 \mathrm{D}$ flow structure in the separation zone, where the gas from the shear layer $S L_{1}$ enters the reverse flow region $Z$ and then leaves this region through the side boundaries. The stream in the separation zone is

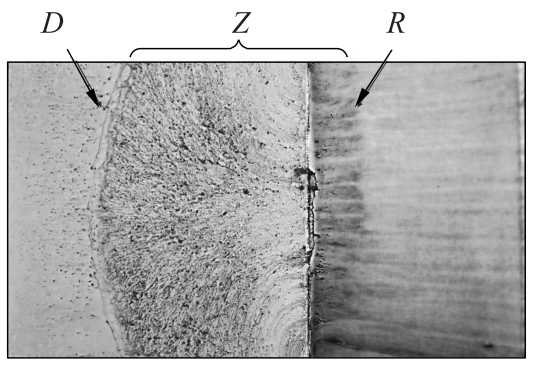

(a)

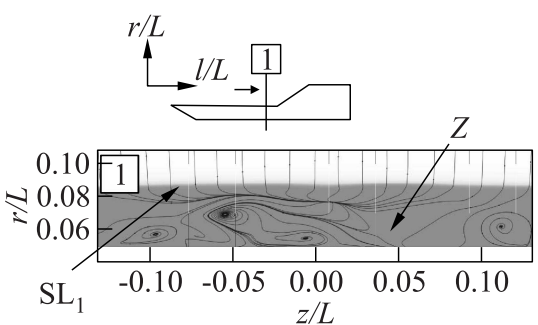

(c)

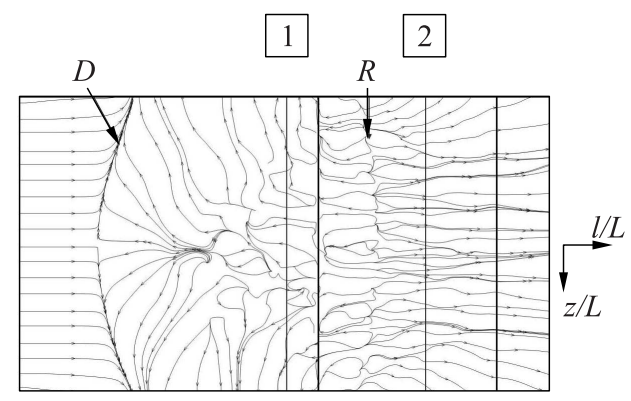

(b)
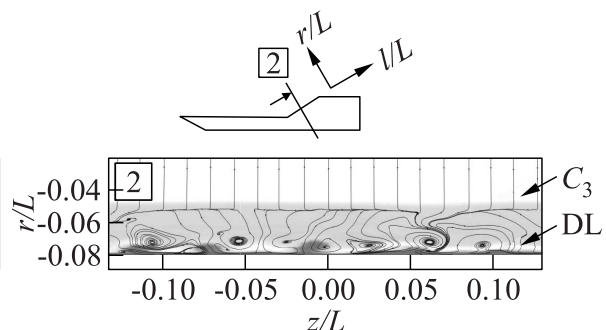

(d)

Figure 5 Streamwise vortices in the compression corner at $\alpha=0^{\circ}$ 
highly turbulized, this observation is evidenced by both oil visualization and numerical data.

Figure $5 c$ shows a fragment of cross section $1(l / L=-0.1)$ located near the longitudinal symmetry axis of the model. The cross section is normal to the horizontal plate. The calculated distribution of the total pressure is shown in the figure. Here, the reverse flow region $Z$ (dark area), the shear layer $\mathrm{SL}_{1}$, and the free stream moving over this layer (light area) are seen. The streamlines are constructed from the normal and transversal components of the flow velocity vector on the plate. Figure $5 d$ shows a similar distribution of the total pressure in cross section $2(l / L=0.58)$ normal to the plane of the ramp surface ( $\mathrm{r}$ is the normal coordinate to the ramp surface). Here, the low-pressure gas region near the model surface (dark area), the high-pressure layer DL (light wavy line), and the free-stream zone above this layer, separated from the free stream by the shock $C_{3}$, are shown. The streamlines are constructed from the normal and transversal components of the flow velocity vector on the ramp surface.

It is seen that both in the interior of the separation zone and in the flow behind the reattachment line, the gas is entrained in vortex motion with generation of streamwise vortices. An experimental description of such vortices in a supersonic separated flow was given in [10], and numerical simulations were reported in [6]. Traditionally, it is believed [2] that the mechanism of origination of such vortices is the same mechanism that underlies the production of TaylorGörtler vortices in subsonic flows. The flow changes its motion direction in the compression corner. The shear layer loses its stability because of the action of the centrifugal forces that arise due to bending of the streamlines [11].

Simultaneously, the present data point to one more possible mechanism of vortex formation (Fig. 6). The vorticity transport equation is [12]

$$
\begin{aligned}
\frac{\partial}{\partial t}\left(\frac{\Omega_{k}}{\rho}\right)+ & u_{j} \frac{\partial}{\partial x_{j}}\left(\frac{\Omega_{k}}{\rho}\right) \\
& =\frac{\Omega_{j}}{\rho} \frac{\partial u_{k}}{\partial x_{j}}+\frac{1}{\rho^{3}} \nabla \rho \times \nabla p+\frac{1}{\rho} \varepsilon_{i j k} \frac{\partial^{2}}{\partial x_{i} \partial x_{j}}\left[\mu\left(\frac{\partial u_{i}}{\partial x_{j}}+\frac{\partial u_{j}}{\partial x_{i}}\right)\right] .
\end{aligned}
$$

This equation includes an additional term $\nabla \rho \times \nabla p$, which describes the barocline effect; namely, the vector of the density and pressure gradients goes to the origin of the vorticity $\Omega$ (vector quantity). Here, $\rho$ is the gas density; $u$ is the velocity; $p$ is the pressure; and $\mu$ is the coefficient of dynamic viscosity.

The reattachment line $R$ is curvilinear near the side edges of the model. The curvature gives a transversal component of the pressure gradient (whose direction is parallel to the model surface and is normal to the stream direction), while the high-pressure layer DL induces a component of the gas density gradient along the normal to the model surface:

$$
\Omega_{k}=\Omega_{i j}=\frac{1}{2}\left(\frac{\partial u_{i}}{\partial x_{j}}-\frac{\partial u_{j}}{\partial x_{i}}\right)
$$




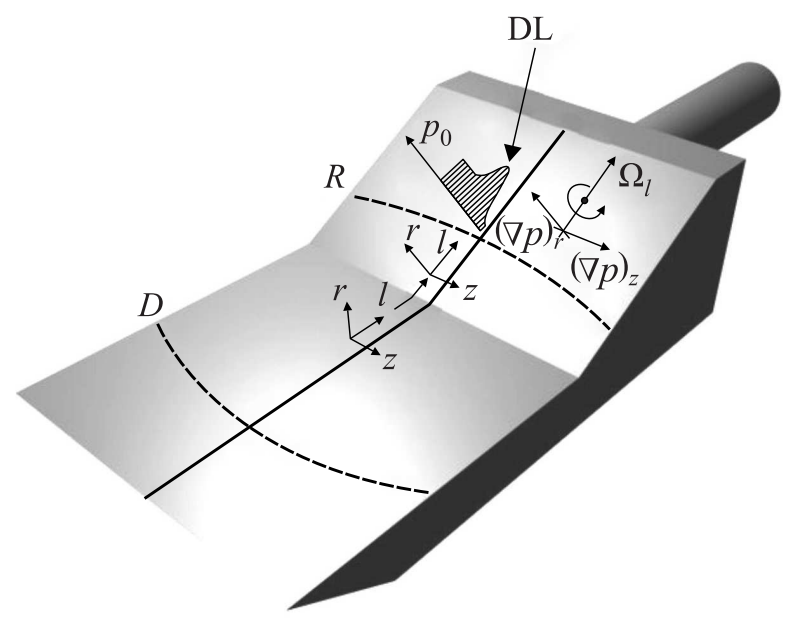

Figure 6 Generation of streamwise vortices near the reattachment line in a supersonic flow near the ramp surface

As a result of the baroclinic effect [13], the streamwise component of the vector of vorticity arises $\left(\Omega_{l}\right)$, and it is aligned in the streamwise direction from the reattachment line $R$. This circumstance also promotes the formation of streamwise vortices, in the regions both below and above the layer DL, and in the interior of the reverse flow region.

\subsection{Wall-pressure Fluctuations Near the Shear-Layer Reattachment Line at Different Angles of Attack}

In the experiments, the wall pressure fluctuations $p^{\prime}(t)$ near the shear layer reattachment line were measured. Since the flow reattachment line is shifted in the upstream direction with increasing angle $\alpha$, the pressure fluctuations near the point $R$ were measured only for $\alpha=0^{\circ}$, whereas the pressure fluctuations for $\alpha=5^{\circ}, 10^{\circ}$, and $15^{\circ}$ were measured at the region downstream of the point $R$.

Figure 7 shows the Fourier spectra of the wall pressure fluctuations $p^{\prime}(t)$ for $\alpha=0^{\circ}, 5^{\circ}, 10^{\circ}$, and $15^{\circ}$ (respectively, $a, b, c$, and $d$ ). Here, the spectral amplitude was normalized by the wall pressure $p_{\operatorname{tr}}$ at the location of the transducer of pressure fluctuations $(l / L=0.18)$. The Strouhal number $\mathrm{Sh}=f L / u_{\infty}$ was calculated from the plate length $L$, the frequency $f$, and the free-stream velocity $u_{\infty}$.

The maxima of the fluctuations marked with arrows 1 and 2 deserve mentioning. At $\alpha=0^{\circ}$ and $5^{\circ}$, maximum 1 was greater in amplitude than maximum 2, 


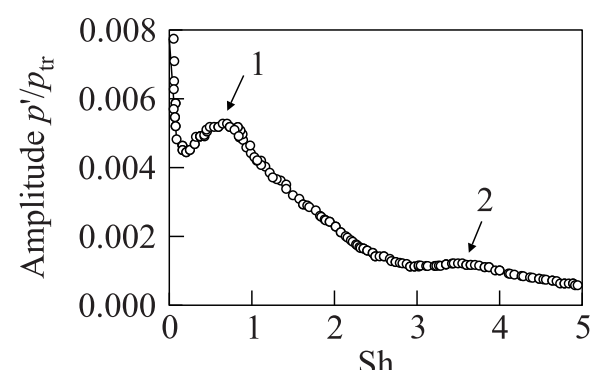

(a)

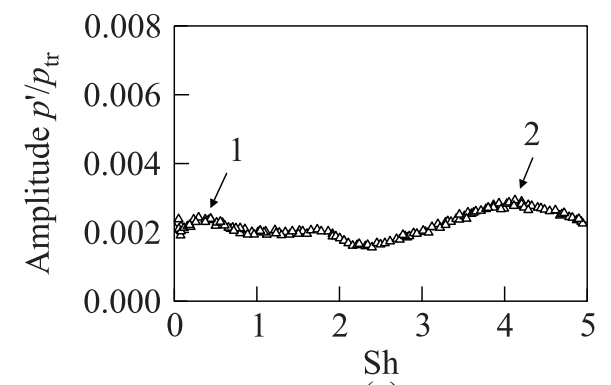

(c)

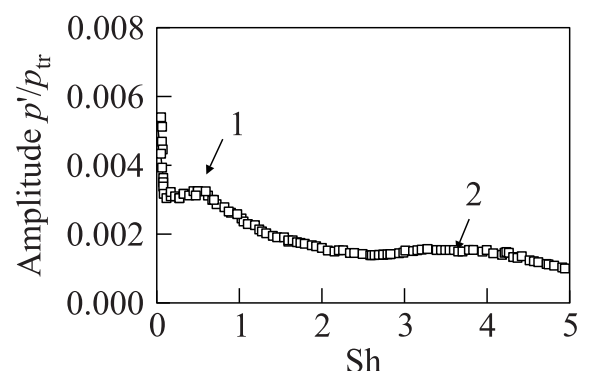

(b)

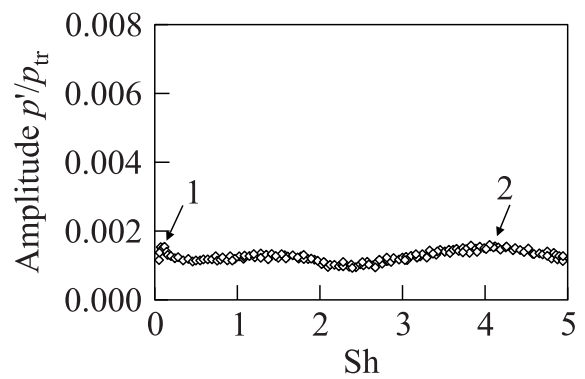

(d)

Figure 7 Spectra of wall-pressure fluctuations for different angles $\alpha$ : $(a) 0^{\circ} ;(b) 5^{\circ}$; (c) $10^{\circ}$; and $(d) 15^{\circ}$

while the opposite relation was observed at $\alpha=10^{\circ}$ and $15^{\circ}$. With increasing angle $\alpha$, the frequency of maximum 1 increases, while the frequency of maximum 2 remains roughly unchanged.

\section{SUMMARY}

For a supersonic flow velocity $\left(\mathrm{M}_{\infty}=6\right)$, the shock wave structure of the compression corner flow and the wall pressure fluctuations in the flow reattachment region for angles of attack $\alpha=0^{\circ}, 5^{\circ}, 10^{\circ}$, and $15^{\circ}$ were studied.

It is shown that a complex configuration of interacting shock waves is formed in the compression corner at high Mach numbers in the flow region above the plate surface. It is shown that the separated flow near the compression corner model has a 3D structure.

The existence of a high-pressure layer (dynamic layer) is found. The dynamic layer is located above the boundary layer in the downstream region from the flow reattachment line. The presence of streamwise vortices is observed. Streamwise vortices are formed near the reattachment line and are located in the downstream 
direction and inside the reverse flow region. A probable cause for the formation of such vortices could be the baroclinic effect due to the existence of a density gradient in the high-pressure layer and a transversal pressure gradient.

It is observed that the spectrum of pressure fluctuations involves two local maxima. For the angles of attack $0^{\circ}$ and $5^{\circ}$, this spectrum is dominated by the first maximum; for the angles of attack $10^{\circ}$ and $15^{\circ}$, the prevalence of the second maximum is observed.

\section{REFERENCES}

1. Chang, P. K. 1970. Separation of flow. Oxford-London-N.Y.: Pergamon Press.

2. Borovoy, V. Ya. 1983. Gas flow and heat transfer at shock waves\&boundary layer interaction zones. Moscow: Mashinostroenie. 141 p. [In Russian.]

3. Graur, I. A., and D.E. Zeitoun. 2003. Supersonic flow around a hollow cylinder flare configuration. EWHSFF Proceedings. Spain, Barcelona. 212-20.

4. Passaro, A., and L. Biagioni. 2003. Hypersonic compression ramp flows: A comparison of DSMC computations and experimental results. EWHSFF Proceedings. Spain, Barcelona. 361-68.

5. Schrijer, F. F. J., F. Scarano, and B. W. van Oudheusden. 2005. Application of PIV in a Mach 7 Ludwieg tube flow facility. 6th Symposium (International) on Particle Image Velocimetry Proceedings. Pasadena, California, USA. $14 \mathrm{p}$.

6. Neiland, V. Ya., L. A. Sokolov, and V. V. Shvedchenko. 2009. Structure of supersonic separated flow past a compression ramp at different values of the temperature factor. Advances in continuum mechanics: Dedication to the 70th Anniversary of academician V.A. Levin. Collection of scientific papers. Vladivostok: Dal'nauka. 540-62. [In Russian.]

7. Babinsky, H., and J. K. Harvey, eds. 2011. Shock wave-boundary-layer interaction. Cambridge University Press. 462 p.

8. Edney, B. 1968. Anomalous heat transfer and pressure distributions on blunt bodies at hypersonic speeds in the presence of a impinging shock. FFA Report No.115. Aerononautical Research Institute of Sweden. 93 p.

9. Hempsh, M. J., and J. N. Nielsen, eds. 1986. Tactical missile aerodynamics. N.Y.: American Institute of Aeronautics and Astronautics, Inc. Vol. 1.

10. Ginoux, J. J. 1971. Vortex system at the downstream region of the high-speed flow reattachment point: An approximate solution. AIAA J. 9(4).

11. Schlichting, H. 1979. Boundary layer theory. New York: McGraw Hill.

12. Smits, A. J., and J.-P. Dussauge. 2006. Turbulent shear layers in supersonic flow. Springer Science, Business Media, Inc. 410 p.

13. Gutmark, E. J., K. C. Schadow, and K. H. Yu. 1995. Mixing enhancement in supersonic free shear flows. Ann. Rev. Fluid Mech. 27:375-417. 